\title{
THE FIVE LAYERS OF THE INTERNET ON THE COMPUTING LEVEL
}

\author{
Bing Li \\ Department of Computer Engineering, \\ Xi'An Technological University, XiAn, China
}

\begin{abstract}
To share huge amount of heterogeneous information, the Internet is reconstructed to consist of five layers, including routing, multicasting, persisting, presenting and humans. Routing layer establishes the fundamental substrate and locates resources with social disciplines. Multicasting layer disseminates data efficiently based on the routing. Persisting layer accesses persistent data with minimum dedicated resources. Presenting layer absorbs users' interactions to adjust the underlying layers through connected views to users. Different from the lower layers, the topmost one is made up with humans, which are social capital dominating the Internet. Within the upgraded infrastructure, besides the situation that a lower layer supports its immediate upper one, the humans layer influences the lower ones by transferring social resources to them. Those resources lead to adaptations and adjustments of the software layers since each of them needs to follow social rules. Eventually, the updated underlying layers return latest outcome to users upon those modifications.
\end{abstract}

\section{KEYWORDS}

Social Computing, World Wide Web, Peer-to-Peer computing, Software Architecture, Social Network Services, Information Sharing, Routing

\section{INTRODUCTION}

This document describes, and is written to conform to, author guidelines for the journals of AIRCC series. It is prepared in Microsoft Word as a .doc document. Although other means of preparation are acceptable, final, camera-ready versions must conform to this layout. Microsoft Word terminology is used where appropriate in this document. Although formatting instructions may often appear daunting, the simplest approach is to use this template and insert headings and text into it as appropriate.

It is believed that the Internet on the computing level should be reconstructed since it has never been designed in a proper way [1]. For its existing downsides, it is tough to share the huge amount of heterogeneous information to the large-scale of heterogeneous users. To upgrade, a five-layered infrastructure is proposed, which includes the routing, the multicasting, the persisting, the presenting and the humans from the bottom to the top, respectively. 
The term of the Internet on the computing level is defined as the Internet-based distributed environment concentrated on resource management and fundamental architectures to support high performance and low cost applications. As a counterpart beneath it, the Internet on the communication level aims to support raw data transmission between two nodes over the Internet. At present, TCP/IP is its primary representative of the level. This paper extends the definition of the computing level in the sense that all of the issues over TCP/IP are included in the scope. To simplify, when mentioning the term of the Internet in the paper, it specifies the one on the computing level.

The routing layer is responsible for managing and locating the tremendously huge resources over the Internet. Until now, a proper routing mechanism is still not available over the Internet. Although Google-like systems take this responsibility and win the huge successes in the industrial domain, they do not touch the essences. It is convinced that the routing over the Internet must follow the rules of social resources [2][3] rather than any other machine-based algorithms.

The multicasting layer is mandatory for the Internet system. To date, the protocol, HTTP (HyperText Transmission Protocol), is still one of the most important solutions to transfer data over the Internet. It aims to transmit text-based or lightweight data. For that, it missed a lot of issues, such as the distributed topology, the transmission environment and the data characteristics, etc. When heavyweight [4] data or timing-sensitive [5] data are to be disseminated, the protocol becomes out of date. It is required to design a bunch of new algorithms based on the routing layer to distribute heavyweight and timing-sensitive data in the high performance and low cost manner.

The third layer is the persisting that supports high performance huge data saving and accessing over the Internet scale with minimum dedicated resources investments. To upgrade, it must be aware that the law of the socialized distributed environment is different from that of traditional machine-based ones. The utilization of undedicated resources [6] that are controlled by their proprietary owners is crucial to the persisting layer. In addition, it is also required to enhance the system with supports from the underlying layers, i.e., the routing and the multicasting.

The presenting layer demonstrates a fundamental application to users. The layer ameliorates the traditional WWW [7] in terms of raising the quality of data connections and supporting upgraded publishing and reading approaches. Moreover, the layer is also an approach to bind its upper layer with the underlying ones together. Traditionally, the WWW [7] plays the role, which aims to connect all of relevant data, i.e., Web pages. However, neither does it provide mandatory management approaches to build the linked world, nor does it guarantee the quality of the connections among pages such that it does not make sense to follow links to keep accessing referenced data.

The topmost layer is the one of humans, which does not exist in any traditional software infrastructures. In a conventional system, all of its subsystems run independently without human interventions, which is different from the dominant effects of the humans layer. The layer not only determines the outcome of the underlying layers but also governs their performance and cost. Its influences are transferred to each of the underlying layers although it owns only one direct link between itself and the routing layer, which is responsible for absorbing and managing social resources from the humans layer. Traditionally, a directed connection exists between two immediate neighboring layers, which play the roles of the source and the destination respectively from the lower to the upper. In contrast, the influences of the humans layer span all of the layers, 
starting from itself, passing through the routing, the multicasting, the persisting, to the presenting finally.

It should emphasize that the paramount value is raised from humans on the Internet rather than any other traditional algorithms. For that, a new computing model is proposed. That is, while the resources of the systems include traditional ones, such as CPU cycles, storage and bandwidth, it is more important to introduce a new kernel to control those resources. The new kernel is the humans layer which provides the most precious value, i.e., the socialized capital [3][4], to the Internet.

The paper consists of the sections to present its primary ideas. Section 2 discusses the previous related researches in the domain and makes concise comparisons. Section 3 summarizes the generic requirements of users over the Internet. Section 4 explains in details about the layers of the Internet on the computing level. Section 5 articulates the collaboration of the layers. Section 6 gives a brief summary about the current and future work.

\section{RELATED WORK}

As Barabasi Albert-Laszlo [1] said researchers would not design the Internet like present if they knew its natures as deeply as nowadays.

\subsection{The Routing}

How to design a routing algorithm that helps each node to find colleagues and computing resources is critical for a large-scale information system over the Internet. Such a system is open, huge, heterogeneous and even unrevealed such that no dedicated coordinators are competent to handle the problem for the system.

\subsubsection{Routing for Publishing}

The goal of routing for publishing is to gain as many readers as possible. However, no any routing answers which users would be potential readers.

The problem is caused primarily by the fact that all of the users over the Internet are not registered uniquely. Moreover, it is tough to identify their interests even though their profiles are available. Some applications [8][9] over the Internet intend to solve the problem. They require users to have an account. Moreover, any node in the systems has followers or friends. Thus, when publishing, those users become the potential readers.

While the systems make progresses, the issue of routing for publishing still remains the old states in the overall infrastructure of the Internet. The exact routing algorithms should be refined by taking into account underlying social capital.

\subsubsection{Routing for Reading}

The questions on the issue of routing for reading are what is the appropriate way to present the huge amount of data to users and how to accomplish it, respectively. 
When the Web was popular in 1990s, a directory service believes that data is exhibited in a hierarchical manner. However, it does not guarantee that the obtained pages meet users' requirements. Additionally, it is difficult to maintain a huge amount of heterogeneous Web sites manually. Even worse, the huge Web can hardly be abstracted to the static hierarchy of URLs upon a limited count of categories.

Then, the keyword searching, such as Google, takes the place of directory services. It aims to show data that matches users' keywords exactly or approximately. The service fulfills one of users' requirements of accessing information. However, it is far from a comprehensive solution. Different from traditional data retrieval approaches [10], it is expected that retrieved results form a particular structure upon their inherent senses rather than one sequence of irrelevant pages. Moreover, the searching consequences rely heavily on keywords from users. Over the Web, the quantity of pages is extremely larger than that of words of natural languages. Thus, Google-like systems work better only when searching by a sequence of keywords existing in pages rather than by a fuzzy term or a sequence of ones to be analyzed. Another problem is that the systems have to match all of indexed pages crawled from the Web and most matching is a waste of cost.

Some social network based systems [8][9] merge to overcome the difficulties. That is, data is presented in a context formed by a reader's friends or followees such that users keep highly active to read information from the context. The topic is tough since it associates with the difficult question, i.e., what on earth a large-scale of heterogeneous and homogenous data is interweaved [11][12]. If it was answered, the proper solution to presenting data could be proposed. The paper

puts forward approaches to do that. Eventually, the solution is applied to the underlying infrastructure of the Internet on the computing level comprehensively in order to support the multicasting, the persisting and the presenting other than users.

\subsection{The Multicasting}

The multicasting is divided into two categories, the messaging and the streaming, according to the data transmission character. If data is transferred in a request/response manner, it belongs to the messaging protocol. Instead, if data is transmitted among nodes in the form of flowing from a source to a destination continuously, it is believed to be a streaming protocol.

\subsubsection{The Messaging Protocol}

The protocol of HTTP transfers data in the messaging way. Through it, the server usually encounters the difficulty to respond clients when receiving a large number of concurrent requests. Even worse, because of the richness of heavyweight data, the cost of the server becomes higher.

It is expected to upgrade the distributed environment and provide high quality routing services to accelerate the messaging.

\subsubsection{The Streaming Protocol}

To transmit heavyweight data, a bunch of competitors [4][5][13] are proposed. They attempt to form a decentralized topology to perform multicasting in a streaming way.

The problems of BitTorrent are found in its low quality routing to find collaborators. It is caused 
by the fact that BitTorrent cannot detect the preferences of multicasting participants, especially the social relationships among them.

To multicast timing-sensitive heavyweight data, BiToS splits data into two sets, a high priority one and a low priority one [5]. Then, it performs BitTorrent algorithms on both of them concurrently. Therefore, although BitTorrent rarest-first algorithm is applied, the playback buffered data from the high-priority set is filled fast enough to become consecutive approximately. The problem of BiToS is the same as that of BitTorrent in terms of the lack of the high quality routing.

Tribler [13] can be regarded as Facebook or Twitter for multicasting. It finds potential collaborators from a buddy list before performing the multicasting with BitTorrent. However, the drawback of TRIBLER is caused by the buddy list as well. While it is better than the random routing of BitTorrent, it does not make sense that a user always has the same preference as its friends.

It should consider the approach to place users into a wider context other than circles of friends. Then, it provides a node with more opportunities to find potential collaborators based on either social relations or common interests. Moreover, it is believed that the routing for multicasting is an interactive and adaptive procedure on the fly other than a one-step task performed before data transmission.

\subsection{The Persisting}

The major problem to persist the huge amount of data over the Internet is to confirm the distinctive principles that determine the performance and the cost.

Cloud computing [14] answers the question by providing sufficient dedicated resources like data centers. It is traditional since it does not consider the natures of the Internet. It is a solution to enterprises rather than the Internet environment.

OceanStore [6] utilizes the undedicated resources from proprietary users like cafeteria owners by attracting them with business incentives. The approach lowers the cost to persist data with the participation of business-oriented resources. With the support of the non-technical design, the performance is raised to a high degree, especially for the popular data.

It is required to borrow the experiences of OceanStore in the persisting layer of the Internet on the computing level with in-depth understanding of the Internet. Social incentives and social capital other than business ones should be taken into in the design of the Internet infrastructure.

\subsection{The Presenting}

The World Wide Web [7] is the fundamental application of the Internet on the computing level. Without the support of an essential underlying infrastructure, the WWW becomes withered such that it has lost its value to present and manage the huge amount of data over the Internet. 
Upon the World Wide Web, an author is authorized the privilege to connect one single term or one phrase inside Web pages to any other ones through the techniques of hyperlinks. Tim-Berners Lee [7] believed that relevant data was weaved together in this way such that readers can follow the links to access the Internet.

Unfortunately, it is seldom for an author to spend sufficient time adding links since it brings additional workload. Additionally, advertisements replace many high quality pages. A reader loses interests to navigate along the routes formed by the links because of that. As a matter of fact, WWW is being degenerated into a graph, which lacks significant connections.

\subsection{Conclusions}

In brief, the current Internet on the computing level was full of drawbacks in terms of its infrastructure. It results in high cost and low performance applications. Moreover, it is also a barrier to fulfill users' data accessing requirements. The Internet on the computing level should be redesigned upon the nature of the Internet to provide publishers and readers with an appropriate environment to access the large information world.

\section{THE INFRASTRUCTURE OF THE INTERNET ON THE COMPUTING LEVEL}

To resolve the problems of the current Internet on the computing level, it is indispensable to establish the New Infrastructure of the Internet on the Computing Level (NIICL), which reflects the natures of the Internet. The system consists of five layers, the routing, the multicasting, the persisting, the presenting and the humans, which are shown in Figure 1.

The term, the computing level, is used to differentiate from the one that supports fundamental communications between any two nodes over the Internet. Rather than the protocol of TCP/IP to exchange data physically, the new infrastructure contains all of the tasks based on sharing data logically.

\subsection{The Differences}

Different from classic layer-based software architectures that work only in the way of a lower layer supporting its immediately upper layer from the bottom to the top, NIICL is an interactive layered system. In the architecture, similar to traditional ones, a lower layer supports its immediately upper one. In addition, the routing layer is also visible to the humans one such that it has to adjust for crucial updates of the topmost layer. Within the fundamental infrastructure, all of the lower ones are under the domination of the topmost, the humans. The influences of the topmost one are transferred directly or indirectly to the underlying ones. For the distinctness of the system, it claims that the Internet should be involved with social resources other than computing resources. That is, such a system relies mainly on humans rather than computers. In another word, the social capital from the humans layer guarantees the quality of the Internet applications.

\subsection{The Humans Layer}

The layer of the humans is made up with all of the users of NIICL. According to their scales, the 
users are divided into individuals and organizations. According to their roles, the users consist of publishers and readers. Users' behaviors online rely only their inherences, preferences, experiences, knowledge and common senses, i.e., human capital [2], which is accumulated during a long term.

The ways to capture the human capital is unavailable yet. For that, it is difficult to detect a user's tastes and intents with their actions directly. As a system to share huge information, it becomes tough to publish and read since it is difficult to find relevant targets, i.e., the readers and the data. Then, it is impractical for them to collaborate with one another and perform the Internet computing efficiently.

Fortunately, rather than human capital, social capital [2][3] or social resources are convenient to be captured. No matter whether to publish or read, users need to interact with others. It is feasible to retain the interactions by the concept of connections or neighbors. With the growth of the system, a huge graph is constructed.

In short, human capital from the humans layer dominates the Internet through users' interactions to access data. Although they are tough to be identified, it is convenient to map human capital to social capital through interactions among users.

\subsection{The Routing Layer}

The routing layer is defined as the mechanism to discern and locate sufficient resources for any other layers to support their activities in a high performance and low cost manner. To do that, it explores the routes between readers and authors. That is why the layer is named the routing. For a large-scale system like the Internet, it is expected that the routes be as short as possible to raise the performance.

One of the primary tasks of the layer is to discern social resources from the topmost, the humans layer. To do that, the concept of a connection is defined as the interaction between an author and a reader. As all of the users of the Internet perform publishing and reading following their preferences, it is time to retain those connections and construct a graph upon those interactions. It indicates that the topmost layer is associated with the bottom one directly, which results in the social graphs distinguished by their node types, data, circles and humans, respectively.

After the graphs are available, it is time to maintain, rank and find relevant resources upon them whenever publishing and reading are performed. The primary principle to do that is to identify those nearby nodes of a publisher or a reader since it is believed that they are the potential resources to fulfill the requirements of the publisher or the reader. That is, they are willing to accept data from the publisher or they hold data that the reader needs.

\subsection{The Multicasting Layer}

The multicasting layer is responsible for transmitting data to potential computing devices, which are located by the routing layer before the transmission is started as well as during the procedure of the transmission.

With respect to the characteristics of social networks [8][9], it is known that a large portion of data is published by an extremely small number of users, i.e., super nodes. Most users play the 
role of readers as ordinary nodes. Therefore, it is a heavy load to send data from one publisher to its readers. The seeking is performed initially before the transmission is started. Once if those resources are available, an appropriate topology is constructed between a publisher and the located resources to balance the load and raise the performance.

In addition, it is critical to keep on routing on the fly since the potential resources are not stable in NIICL. All of resources participating in the transmission belong to proprietary users. Then, they join and leave the topology casually during the multicasting procedure. To lower the impacts of the departure, it is required to place those devices into a fault-tolerant graph rather than a tree. It is also necessary to keep seeking potential resources by interacting with the underlying routing layer during the entire procedure.

\subsection{The Persisting Layer}

The persisting layer aims to save huge amount of data into mass storage devices. The first problem is whether storage resources are dedicated or not. As a storage system for the Internet, it is impossible to provide sufficient dedicated resources because of the large scale. A reasonable system is made up with both dedicated and undedicated storage resources. It is critical to find undedicated resources from proprietary users, which are original publishers as well as forwarders. They would like to contribute their resources.

The next issue is where to persist. As a global system, the locations of data are critical to the performance. Since consumers of public data reside each corner of the globe, it is tough to answer the question. Fortunately, it is unnecessary to care about the issue because of the remarkable routing layer. Since readers might become potential resource providers such that their physical locations are identical to where the data should be persisted. For the nature of the routing layer, new users gain high performance from the previously persistent data. In the solution, physical locations are hidden by social positions [15], i.e., the social capital, to a great extent.

The final problem is how many replicas to persist. The number of data replicas is another critical factor to determine the performance. It is also difficult to estimate the number for the Internetbased storage system with traditional approaches. To resolve the problem, data is replicated and persisted to the owners who are willing to share with the assistance of the routing layer. During the procedure, the number of replicas is controlled by its original publisher and secondary publishers. The count of replicas depend on the popularity of data. In essence, the quality of data determines its quantity of replicas. Thus, it is unnecessary to take into account the issue either when persisting.

\subsection{The Presenting Layer}

The presenting layer is responsible for interacting with users and showing data to users. The layer aims not only to fulfill users' requirements but also to achieve the goal to cohere users with the system. Through the interactions, users become the dominators to guide the underlying layers to perform, adjust and optimize. It is the layer that converts human and social capital to govern the entire system. Its value is different from traditional presentations which separate users from computing systems and leave all of tasks to machines.

While the infrastructure is governed by the social graphs of data, circles and humans, they are updated on the fly following users' behaviors through the layer. Those behaviors consist of two 
types, i.e., writing and reading. Both of the behaviors result in the connections for data, circles and humans. In brief, they are collected by the presenting layer and transferred to the underlying ones.

\section{THE COLlabORATION OF THE LAYERS}

The five layers form the new infrastructure of the Internet on the computing level (NIICL). They works in the traditional way the lower layer supporting its immediately upper one from the bottom to the top as well as they interact with one another controlled by social capital [15]. The collaboration among the layer is illustrated in Figure 1.

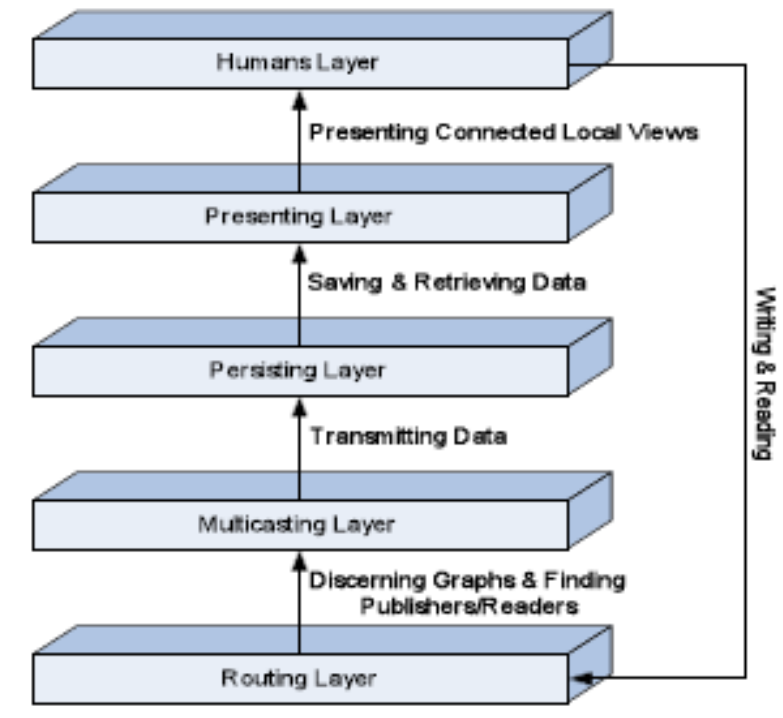

Figure 1. The Collaboration of the Five Layers

\subsection{The Supporting From the Bottom to the Top}

The routing layer supports its immediately upper one, the multicasting, through locating sufficient numbers of readers and publishers to publish data in a large scale. Moreover, a reader obtains accurate and comprehensive responses from relevant publishers and other readers.

The multicasting layer plays the crucial role to transmit data in a high quality way to support the persisting layer. Only if data is disseminated rapidly, the services of persisting data are performed efficiently in a large scale heterogeneous environment.

The persisting layer supports the presenting layer by keeping data in a proper manner in terms of nearby locations and rational replicas. That is the bedrock to present data in a high performance and low cost way. Although users and data are located in various physical places, both of them are presented in social locations.

The presenting layer shows connected data, circles and humans to users of the humans layer. It hides the underlying mechanism from users such that users gain a comfortable data accessing environment that matches users' personal preferences and social cognition. 


\subsection{The Interactions Among the Layers}

The distinctive character of NIICL is that those layers interact with each other rather than support immediately upper layers only. It is caused by the core resources of the Internet on the computing level, i.e., human and social resources. The social graphs are always ameliorated by user behaviors in the humans layer, which is the dominant component of the infrastructure.

When users enter the system initially, they become members of the humans layer. Through the presenting layer, it is convenient to observe and locate the connected data, circles and humans they are interested in. Their behaviors are supported efficiently and effectively through those views. More important, it results in the updates of the connections surrounding them such that it impacts the consequences from the routing layer directly. Thereafter, the relevant multicasting and the persisting are improved for both of them depending on the routing. It is no doubt that it also results in updated connected views presented to users.

\section{THE CURRENT AND FUTURE WORK}

The research has been carried out for more than twelve years since November 2005. The research attempts to convert the existing WWW to the new infrastructure. During the procedure of the research, it is found that the critical problems over the Internet on the computing level are associated with diverse domains, such as social cognition, social network analysis, economics, statistical physics, library \& information science, social computing and statistics, etc. The overall architecture is an important progresses to the current Internet.

As a huge system to share information, it is mandatory to place data in an appropriate form for publishers and readers. From my point of view, the traditional approaches do not fit in the complicated situation of the Internet. During the past years of the Internet development, the trend is apparent. That is, traditional Web sites, directory sites and even search engines are not as significant as social networks oriented systems even in the domain of information sharing. Information structures have innate ties with social capital. It is required to be clarified in the future research.

\section{ACKNOWLEDGEMENTS}

This research is sponsored by the Ministry of Education, Shaanxi Province, China. The number of the support is 14JK1358. I appreciate the support!

\section{REFERENCES}

[1] Albert-laszlo Barabasi. 2014. Linked: How Everything Is Connected to Everything Else and What It Means for Business, Science, and Everyday Life. Publisher: Basic Books, ISBN-10: 0465085733, ISBN-13: 978-0465085736.

[2] Yilei Shao. 2007. Exploring Social Networks in Computing Systems. PhD Dissertation, Princeton University, 2007.

[3] James S. Coleman. 1988. Social Capital in the Creation of Human Capital. American Journal of Sociology, 1988, Volume 94 Supplement, Pages: 95-120. 
[4] Bram Cohen. 2003. Incentives Build Robustness In BitTorrent. 1st Workshop on the Economics of Peer-2-Peer System, 2003.

[5] Aggeglos Vlavianos, Marios Iliofotou and Michalis Faloutsos. 2006. BiToS: Enhancing BitTorrent for Supporting Streaming Applications. Proceedings of 25th IEEE International Conference on Computer Communications, 2006, Pages: 1-6.

[6] John Kubiatowicz, et al. 2000. OceanStore: An Architecture for Global-Scale Persistent Storage. ACM SIGPLAN Notices, Volume 35 Issue 11, Nov. 2000, Pages: 190-201.

[7] W3C. 2000. World Wide Web Consortium. http://www.w3.org.

[8] Matei Ripeanu. 2001. Peer-to-Peer Architecture Case Study: Gnutella Network. Proceedings of 1st International Conference on Peer-to-Peer Computing, 2001, Pages: 99-100.

[9] Ian Clarke, Oskar Sandberg, Brandon Wiley and Theodore W. Hong. 2001. Freenet: A Distributed Anonymous Information Storage and Retrieval System. International Workshop on Designing Privacy Enhancing Technologies: Design Issues in Anonymity and Unobservability, 2001.

[10] Christopher D. Manning, Prabhakar Raghavan and Hinrich Schutze. 2008. Introduction to Information Retrieval. Cambridge University Press, 2008, ISBN: 978-0-521-86571-5.

[11] Mark Granovetter. 1983. The Strength of Weak Ties. American Journal of Sociology 78, 1983, Pages: 1360-1380.

[12] Ronald S. Burt. 1992. Structural Holes: the Social Structure of Competition. Harvard University Press, 1992, ISBN: 0-674-84371-1.

[13] J. A. Pouwelse, et al. 2008. Tribler: A Social-Based Peer-to-Peer System. Journal of Concurrency and Computation: Practice \& Experience - Recent Advances in Peer-to-Peer Systems and Security (P2P 2006), Volume 20, Issue 2, February 2008, Pages: 127-138.

[14] Qusay Hassan. 2011. Demystifying Cloud Computing. Journal of Defense Software Engineering, 2011 (January/February), Pages: 16-21.

[15] Stephen P. Borgatti, Ajay Mehra, Daniel J. Brass and Gluseppe Labianca. 1994. Network Analysis in the Social Sciences. Science, 1994, 323(5916): 892-895.

\section{AUTHORS}

Bing $\mathbf{L i}$ is a faculty of Xi'An Technological University. I have been working here for four years since 2013. Before that, I worked as an associate professor for Peking University, the most famous university in China between 2005 and 2013. I got my $\mathrm{PhD}$ degree in the area of software engineering from Arizona State University in 2004. Moreover, I have some industrial working experiences in Lucent and IBM. He focused on the research in the area of the system-level software for Internet. He proposed the upgraded WWW and tried to program the entire system until now. Because of his rich experiences of programming on the

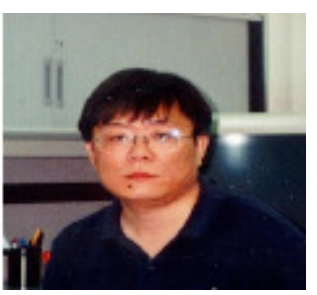
system-level of the Internet, he summarizes a series of APIs and design patterns for large scale distributed system programming, which is called the Great Free Cloud Programming Environment. His personal blog is located at http://greatfree.lofter.com. 